International Journal of Engineering \& Technology, $7(2.21)(2018) 342-344$
WPC

\title{
Interference-based topology control algorithm for noise removal and delay-constrained mobile ad hoc networks using clustering technology
}

\author{
S. Kiruthika ${ }^{1}$, N. Iswarya ${ }^{2}$, A. Sartiha ${ }^{3 *}$, K. Kalaivani ${ }^{4}$, K. Ulaga Priya ${ }^{5}$ \\ ${ }^{1}$ Department of Computer Science \& Engineering, Vels Institute of Science, Technology \& Advanced Studies(VISTAS), Chennai, India. \\ ${ }^{2}$ Department of Computer Science \& Engineering, Vels Institute of Science, Technology \& Advanced Studies(VISTAS), Chennai, India. \\ ${ }^{3}$ Department of Computer Science \& Engineering, Vels Institute of Science, Technology \& Advanced Studies(VISTAS), Chennai, India. \\ ${ }^{4}$ Department of Computer Science \& Engineering, Vels Institute of Science, Technology \& Advanced Studies(VISTAS), Chennai, India. \\ ${ }^{5}$ Department of Computer Science \& Engineering, Vels Institute of Science, Technology \& Advanced Studies(VISTAS), Chennai, India.
}

*Corresponding author E-mail:saritha.se@velsuniv.ac.in

\begin{abstract}
MANET is ad hoc network which is in the top layer of the network. This ad hoc network are connected by mobile devices with flexible topology network to control the delay constrain in the mobile ad hoc network for noise removal in network. The transmission power is managed to minimize the interference taking into account the topology control algorithm also accounts the delay constraint and finds the duplicate data.
\end{abstract}

Keywords: MANET, NS2, AODV, QOS, ROUTING PROTOCOL, ITCD.

\section{Introduction}

The topology control is mostly used in wireless ad hoc network where topology construction and maintenance preserve the connectivity and coverage . The main aim of topology control in the domain is to save energy reduce interference network and it will adjust the transmission security attack to minimize interference delay and cryptographic technique .The administrator has no control over the design of the network specially when the location of the network is random while it deployed in the initial topology.

The source nodes has some control parameters in the network: The power of the node in transmission of data reduces, state and role of nodes: nodes is in active state or sleeping state, gateway and cluster head these are the parameters where the source nodes can change to increase the transmission in the topology of the network.

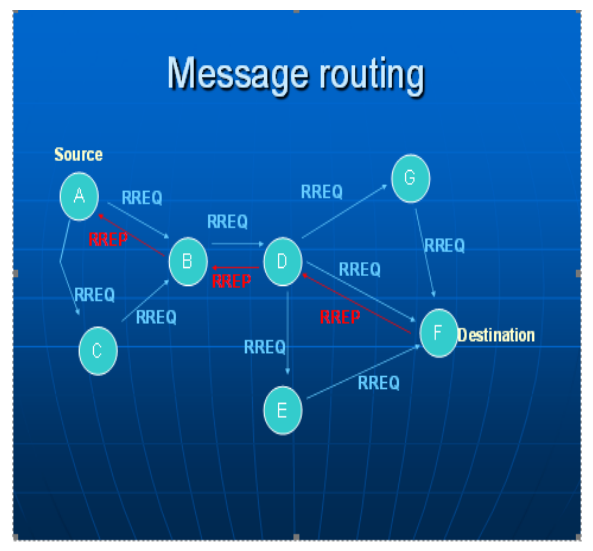

\section{Related work}

Interference-based topology control algorithm for delay-constrained mobile ad-hoc network

This topology network mainly concentrate on the delay of the packet delivery in the network it also measures the quality of the service that received at destination that increases the traffic load in the network this is accessed from end to end network. Packet collision is accounted by connecting with the minimized transmission power ITCD take cares of this connectivity. The topology remove the unstable links which is considered by the mobility, mobility impact with the end to access delay nodes in a path. In mobile ad-hoc network it consider the delay caused by the mobility of nodes.

DISADVANTAGE:

\begin{tabular}{|l|l|}
\hline LOWER & HIGHER \\
\hline $\begin{array}{l}\text { The nodes with lower mobility } \\
\text { can ignored or reduce the delay } \\
\text { in the node }\end{array}$ & $\begin{array}{l}\text { Here the source node data is } \\
\text { transmitted quickly where the link is } \\
\text { unstable and it break }\end{array}$ \\
\hline
\end{tabular}

Scheduling for end-to-end deadline constrained traffic with reliability requirements in multihop networks

Scheduling alone is described, This algorithm describes the problem of designing a constrained traffic network with a policy of end to end deadline in a multi-hop network. It describes three algorithm and characteristics, here it find the scheduled number of continuous nodes that follow each other and the time slots are framed in group. This frame are assumed to have packets arrive at the beginning and empire at end. This model extends the network and traffic model to multiple hop with multiple flows. The packets serves independently by alternative strategy, FIFO and LIFO. 
Throughput and delay in cooperative wireless networks with partial infrastructure

This algorithm can be applied only when the source and destination are in the same cell. It works on the MIMO technology. Hybrid wireless network accounts a scale where number of nodes and number of base stations are same.

\section{Capacity and delay of hybrid wireless broadband access networks}

In a pure wireless $\mathrm{AD}-\mathrm{HOC}$ network it consists of $\mathrm{N}$ number of nodes which cannot satisfy the broadband where the number of users are in a $\mathrm{N}$ number throughput level diminishes, since the optical network are too costly to act .

ADVANTAGE

- throughput capacity

\section{DISADVANTAGE}

- $\quad$ Packet delay

The network faces a drawback if they are loosely coupled when it comes to ad-hoc network tending to see the worst aspects of things. In wireless ad-hoc networks cannot satisfy when the number of node increases in broad band exactly similar traffic is applied with two-dimensional square hybrid wireless networks.

\section{Delay-guaranteed cross-layer scheduling in multihop wireless networks}

The algorithm assures with a finite buffer sizes. It focuses on joint congestion control and scheduling problems, routing in a multi hop wireless networks. But here only congestion control is concentrated more than routing and scheduling. A cross layer framework acquires the optimal throughput without any delay for a multi hop wireless network which is built finally.

\section{RREP messages}

The RREP packet contains for destination address, source, destination sequence number, lifetime and hop count. The RREP send the acknowledgement to the source node when it is reached to the destination node. RREP is itself the destination node since they send an acknowledgement and they act as active route.

Via multiple RREP packets will be transmitted to nodes on the reverse path only if it has more than one node and a valid route.

When the RREP bear back to the source node the intermediate node will update their routing table.

A node will not retransmit the RREP packet if the destination sequence number is lower than the one which is stored in the routing table.

The RREP packet will be retransmitted from the node only if the destination sequence number is higher than the one which is stored in the routing table to decrease the bandwidth use without any quality results. The source receives an RREP packets and it can only and it can act only once.

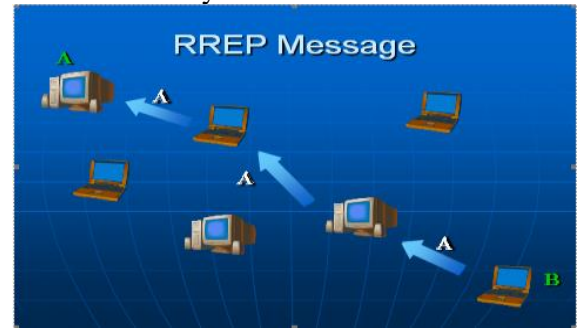

\section{RREQ messages}

Routing request packets broadcasting new path .RREQ has both source and destination with the sequence number address and hop count.
The RREQ request can be uniquely identify broadcast ID and source address.

If the packet does not contains RREQ the destination node drop the packet and then it checks the path whether it has a valid route to the destination.

A route is accounted to be valid only when the destination sequence number is great in number than the present RREQ packets.

RREQ packets and rebroadcast are incremented when it does not contain such kind of route.

If a node contains a valid route to the destination RREP will reply to the source node.

\section{RRER messages}

This message is transmitted when there is a breakage in the link where the node send a message to the source node and it also transfer the message when it is received from some other node also.

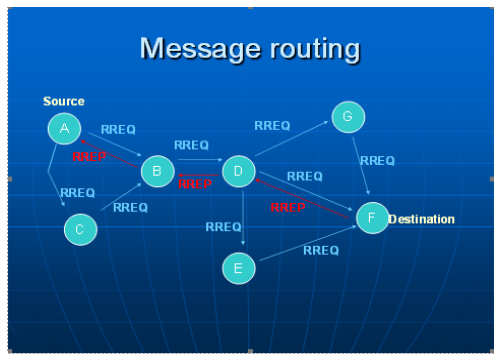

\section{ADHOC network}

AD-HOC network is decentralized infrastructure less network route helps to transfer data easily in a localized network. Where in a AD-HOC network, it use mobile as a connecting device. It get connected through mobile device to transfer data from one node to other, So, here in this design we use this wireless a-hoc network(mobile) it access the mobile device. They, use sequence number to get connected with the $\mathrm{N}$ number node.

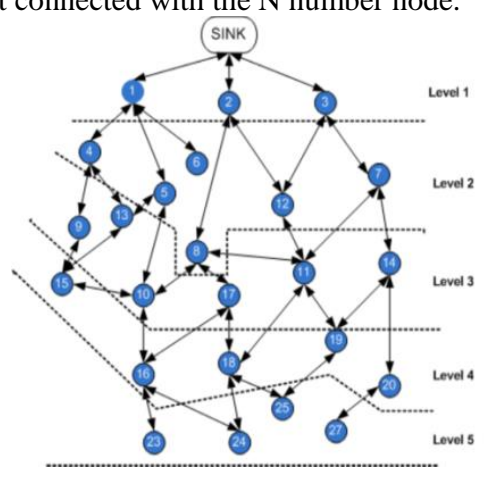

\section{Algorithm}

Ad hoc network routing algorithm mainly work on mobile ad hoc networks. Ad hoc network can perform unicast and multicast routing. Here the decision making is done by the source node. The source node also decide when to disconnect the route path. As ad hoc network performs multicast routing that consist of group member where the group nodes needs to be connected with the source node. Ad hop network and can be connected with the $\mathrm{N}$ number of mobile nodes. Since they have a sequence number.

\section{Hybrid algorithm applied in the network}

HRTC is based on the fact where the conditions can change dynamically in wireless sensor networks. It is impossible to exist in a looped network. But the network can be disconnected when there is a network issue 


\section{System architecture}

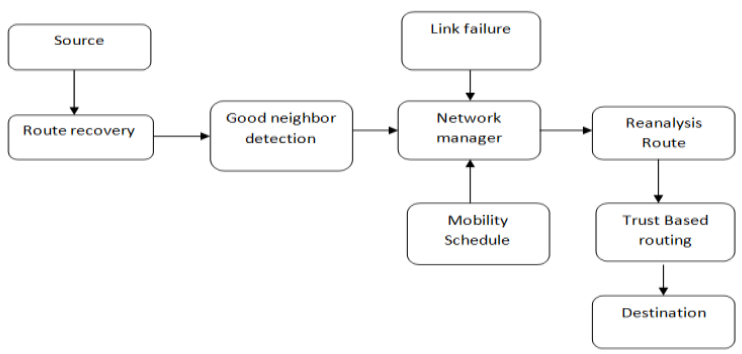

Congestion is one of the major problem which affects all kinds of networks mainly in wireless sensor network. It affects the performance of the network. The traffic of resource control has been solved by congestion control algorithm, Congestion are mitigated and data rate is limited alternative paths are placed to avoid congestion regions by resource control.

\section{Performance measures}
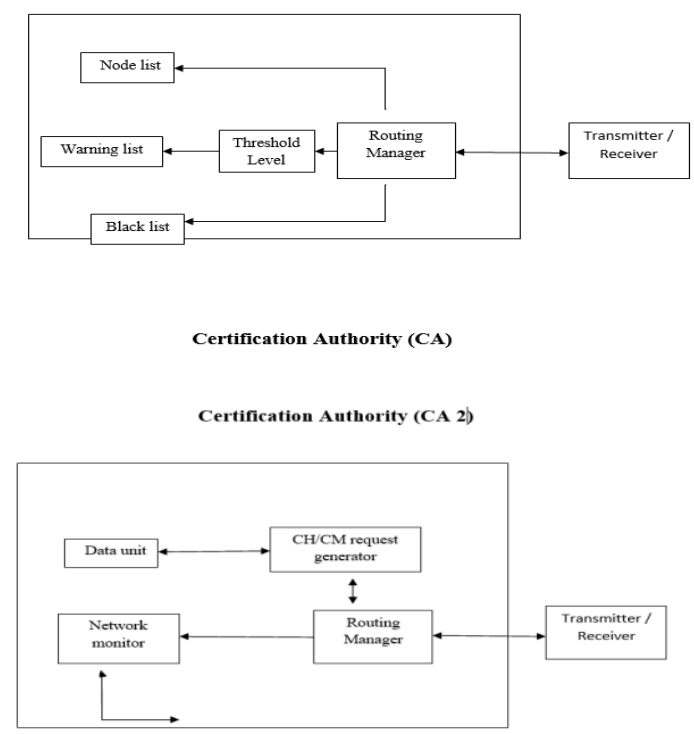

Here in this design node works as both source and destination node source node send the data to neighbor node and it manages the routing path, it maintain a threshold level which is decided by the source node with node list, warning list and black list. Routing manager send request to $\mathrm{ch} / \mathrm{cm}$ request and the data path is send to data unit and network monitor.

\section{Conclusion}

The main purpose of this design to remove the noise removal and delay constraint in the network. The main aim of interference based topology control algorithm is to minimize the interference in the network when the number of nodes increases it also find the duplicate data source node decide how long to hold the network. AODV additionally work on multicast where the group members are maintained by sequence number. Thus the transmission power is increased and the interference is minimized therefore noise level and delay constrained is reduced by using clustering technology.

\section{References}

[1] Zhang XM, Zhang Y, Yan F \& Vasilakos AV, "Interference-based topology control algorithm for delay-constrained mobile ad hoc networks", IEEE Transactions on Mobile Computing, Vol.14, No.4, (2015), pp.742-754.
[2] Xue DY \& Ekici E, "Delay-guaranteed cross-layer scheduling in multihop wireless networks", IEEE/ACM Trans. Netw., Vol.21, No.6, (2013), pp.1696-1707.

[3] Li RG \& Eryilmaz A, "Scheduling for end-to-end deadline constrained traffic with reliability requirements in multihop networks", IEEE/ACM Trans. Netw., Vol.20, No.5, (2012), pp.1649-1663.

[4] Zhu X, Li P, Fang Y \& Wang Y, "Throughput and delay in cooperative wireless networks with partial infrastructure", IEEE Trans. Veh. Technol., Vol.58, No.8, (2009), pp.4620-4627.

[5] Li P, Zhang C \& Fang Y, "Capacity and delay of hybrid wireless broadband access networks", IEEE J. Sel. Areas Commn., Vol.27, No.2, (2009), pp.117-125.

[6] Sharma G, Mazumdar R \& Shroff NB, "Delay and capacity tradeoffs in mobile ad hoc networks: a global perspective", IEEE/ACM Trans. on Networking, Vol.15, No.5, (2007), pp.981992.

[7] Jia XH, Li DY \& Du DZ, "QoS topology control in ad hoc wireless networks", Proc. IEEE Infocom, (2004), pp.1264-1272.

[8] Chou CF \& Suen HP, "Topology-control-based QoS routing (TLQR) in wireless ad hoc networks", Proc. IEEE PIMRC, (2006), pp.1-5.

[9] Mammen J \& Shah D, "Throughput and delay in random wireless networks with restricted mobility", IEEE Trans. On Information Theory, Vol.53, No.3, (2007), pp.1108-1116.

[10] Zhang XM, Zou FF, Wang EB \& Sung DK, "Exploring the dynamic nature of mobile nodes for predicting route lifetime in mobile ad hoc network", IEEE Trans. On Vehicular Technology, Vol.59, No.3, (2010), pp.1567-1572. 